\title{
Scaling up integrated primary mental health in six low- and middle-income countries: obstacles, synergies and implications for systems reform
}

Inge Petersen, André van Rensburg, Fred Kigozi, Maya Semrau, Charlotte Hanlon, Jibril Abdulmalik, Lola Kola, Abebaw Fekadu, Oye Gureje, Dristy Gurung, Mark Jordans, Ntokozo Mntambo, James Mugisha, Shital Muke, Ruwayda Petrus, Rahul Shidhaye, Joshua Ssebunnya, Bethlehem Tekola, Nawaraj Upadhaya, Vikram Patel, Crick Lund and Graham Thornicroft

\section{Background}

There is a global drive to improve access to mental healthcare by scaling up integrated mental health into primary healthcare (PHC) systems in low- and middle-income countries (LMICS).

\section{Aims}

To investigate systems-level implications of efforts to scale-up integrated mental healthcare into PHC in districts in six LMICs.

\section{Method}

Semi-structured interviews were conducted with 121 managers and service providers. Transcribed interviews were analysed using framework analysis guided by the Consolidated Framework for Implementation Research and World Health Organization basic building blocks

\section{Results}

Ensuring that interventions are synergistic with existing health system features and strengthening of the healthcare system building blocks to support integrated chronic care and tasksharing were identified as aiding integration efforts. The latter includes (a) strengthening governance to include technical support for integration efforts as well as multisectoral collaborations (b) ring-fencing mental health budgets at district level; (c) a critical mass of mental health specialists to support task-sharing; (d) including key mental health indicators in the health information system; (e) psychotropic medication included on free essential drug lists and (f) enabling collaborative and communityoriented PHC-service delivery platforms and continuous quality improvement to aid service delivery challenges in implementation.

\section{Conclusions}

Scaling up integrated mental healthcare in PHC in LMICs is more complex than training general healthcare providers. Leveraging existing health system processes that are synergistic with chronic care services and strengthening healthcare system building blocks to provide a more enabling context for integration are important.

\section{Declaration of interest}

None.

\section{Keywords}

Mental health services; integrated care; primary healthcare; low -and middle-income countries; global mental health.

\section{Copyright and usage}

(C) The Royal College of Psychiatrists 2019. This is an Open Access article, distributed under the terms of the Creative Commons Attribution-NonCommercial-NoDerivatives licence (http://creativecommons.org/licenses/by-nc-nd/4.0/), which permits noncommercial re-use, distribution, and reproduction in any medium, provided the original work is unaltered and is properly cited. The written permission of Cambridge University Press must be obtained for commercial re-use or in order to create a derivative work.
The past decade has seen substantial advances in building the evidence base for the effectiveness of task-sharing interventions to close the treatment gap for mental disorders in low- and middleincome countries (LMICs). The World Health Organization's (WHO) Mental Health Gap Action Programme (mhGAP), now in over 100 countries, ${ }^{1}$ and Disease Control Priorities for mental, neurological and substance use disorders, ${ }^{2}$ have played a leading role in these endeavours. In addition, there have been a number of trials on task-sharing of psychosocial interventions to non-specialist health workers in middle-income countries and, to a lesser extent, in low-income countries. ${ }^{3}$ Scaling up of these interventions - most prominently, the integration of mental health into primary healthcare (PHC) in real-world settings - is the next frontier. The challenge in expanding access to care is therefore less about what to implement, but more about how to implement at scale. ${ }^{4}$ Implementation research is a critical vehicle to understand challenges and processes that have an impact on the scalability of integration packages of care in real-world settings.

This paper reports on findings from the Emerald (Emerging mental health systems in low- and middle-income countries) research consortium, involving six LMICs in sub-Saharan Africa and South East Asia (Ethiopia, India, Nepal, Nigeria, South Africa and Uganda) focused on examining health system strengthening needed to support integrated mental healthcare. ${ }^{5}$ The specific aim of this study was to investigate the obstacles, synergies and implications of efforts to scale-up integrated mental health into PHC in district sites in these LMICs. In Nigeria, these integration scale-up efforts were undertaken through a project involving training of primary care providers in the mhGAP Intervention Guide (mhGAP-IG). In the remaining countries the scale-up efforts were through the PRIME (PRogramme for Improving MEntal healthcare) research consortium, which aimed to develop, implement, evaluate and scale-up integrated packages of care for priority mental disorders.

\section{Method}

\section{Settings}

The characteristics of the six countries has been described elsewhere and are summarised in Table $1 .^{6}$ There is wide variation in terms of size, gross domestic income and human development, although there are similarities in terms of governance systems (all countries are democratic republics). None of the countries had a dedicated mental health 


\section{Country and salient features}

Ethiopia Population: 94 million; HDI: 0.448; mental health as a \% of national health budget: $0.9 \%$; psychiatrists/ 100000 population: 0.58 ; psychiatric beds $/ 100000$ population: 0.06

India Population: 1.3 billion; HDI: 0.624; mental health as a $\%$ of national health budget: $0.06 \%$; psychiatrists/ 100000 population: 0.07; psychiatric beds/100 000 population: 1.46

Nepal Population: 28.4 million; HDI: 0.558; mental health as a \% of national health budget: 0.17; psychiatrists/ 100000 population: 0.13; psychiatric beds/100 000 population: 1.0

Nigeria Population: 180 million; HDI: 0.527; mental health as a \% of national health budget: 3.3; psychiatrists/ 100000 population: 0.1; psychiatric beds/100 000 population: 1.3

South Africa Population: 55 million; HDI: 0.666; menta health as a \% of national health budget: 4.0; psychiatrists/100 000 population: 0.28 ; psychiatric beds/100 000 population: 18.0

Uganda Population: 34.6 million; HDI: 0.493; mental health as a \% of national health budget: 0.9 ; psychiatrists/ 100000 population: 0.09; psychiatric beds/100 000 population: 2.77

\section{Description of intervention in the scale-up site(s)}

PRIME intervention. mhGAP training for PHC workers (nurses and health officers), establishing a zonal advisory board with designated mental health coordinators in each district and ensured supply of psychotropic medication, technical support from the PRIME team and regular clinical supervision by a psychiatric nurse. A registration book was introduced to allow routine information monitoring and facilitate detection of drop-out from care and initiation of outreach by community health extension workers

PRIME intervention. mhGAP training for medical officers in community health centres and civil hospitals to deliver pharmacological treatment. Nurses were trained to screen and provide psychosocial intervention packages including healthy activity programme for depression (HAP), counselling for alcohol problem (CAP) and psychoeducation for psychosis. The HMIS system in the PRIME implementation sites was also adapted for the scale-up sites. The PRIME team provided technical support and supervision to the scale-up phase

PRIME intervention. mhGAP training for prescribers (health assistants and medical officers) and psychosocial support along with the HAP and CAP programme for non-prescribers (auxiliary nurses and midwives). The female community health volunteers (FCHVs) received training on the community informant detection tool and home-based care. The PRIME intervention followed a district mental healthcare plan at three levels. At community level, a community awareness programme was conducted by FCHVs and psychosocial counsellors, case detection and referra to health facilities along with follow-up and home-based care was also conducted by FCHVs. At the health facilities level, the prescribers trained in mhGAP provided medical treatments and non-prescribers provided emotional/psychosocial support along with HAP and CAP counselling. A psychiatrist from the district hospital provided supervision to the prescribers and was also the point of referral. Supervision to non-prescribers and a referral counselling service was provided by psychosocial counsellors from TPO Nepal

mhGAP-IG training was conducted for all the community health workers (consisting of nurses, community health officers and community health extension workers) in the intervention PHCs. Engagement and intervention procedure workshops were held with the facility managers and supervisory physicians. The training and workshops focused on identification, treatment (especially psychosocial treatment), and effective use of established referral pathways. Advocacy activities and meetings were conducted with senior policymakers, in particular with the Director of the Oyo State Primary Health Care Board to facilitate the cooperation of the frontline clinical staff and also to ensure that trained staff were not transferred away. The HMIS system was also improved through the collection of additional information that was fed into the HMIS of the state

PRIME intervention. The introduction of a collaborative stepped-care package for chronic care patients with comorbid common mental disorders. Training of PHC nurses in identification of common mental disorders was enhanced through the addition of a mental health module using mhGAP guidelines to the standard Department of Health training in integrated chronic care guidelines called Adult Primary Care. Referral pathways were also strengthened; with facilitybased lay counsellors trained to provide manualised counselling for patients with chronic conditions with mild to moderate depressive symptoms; and doctors oriented to the need to provide medication for those with moderate-severe depression. A task-shared communitybased psychosocial rehabilitation programme facilitated by auxiliary social workers was also introduced for stabilised patients with schizophrenia receiving ongoing medication for symptom management from the PHC facilities

PRIME intervention. Aimed at governance, health facility and community levels. At the governance level, the PRIME team engaged the health managers to ensure buy-in so as to support the integration programme. They were sensitised to mobilise the necessary human and financial resources. At the health facility level, the PRIME team together with the national mhGAP training team trained nurses in assessment and treatment of the priority mental health disorders (psychosis, depression, alcohol use disorder, epilepsy and common mental illnesses) using adapted mhGAP intervention guidelines. The PRIME team also facilitated a district supervision support programme to lower-level health facilities that was carried out by the clinical and nursing officers. Support was also offered to improve the accuracy of the HMIS. At the community level, PRIME facilitated outreach programmes through village health teams (providing basic facts about the common mental health conditions in the area, symptoms, basic management and places of help), as well as the formation of carer and user support groups that were instrumental in reducing stigma in addition to reducing clinical attendances drop-out budget, with South Africa, being an upper-middle-income country, having the most mental health resources per 100000 population. ${ }^{6}$

\section{Intervention characteristics}

All six countries were engaged collaboratively with the respective ministries of health in interventions to integrate mental health into PHC at district level. The main vehicle for integration was training of general PHC providers in the mhGAP-IG, or adapted versions of mhGAP-IG. ${ }^{7}$ The mhGAP-IG provides guidelines for the identification and management of mental, neurological and substance use disorders, including the initiation of psychotropic medication as well as evidence-based psychosocial interventions such as cognitive-behavioural therapy/interpersonal therapy for depression 
and brief motivational interviewing for substance use disorders in non-specialist health settings.

The countries involved in the Emerald study focused on the following priority disorders: depression, alcohol use disorder, severe mental disorders and epilepsy. There was variation across the country sites in relation to: (a) the priority disorders included in the integration efforts; (b) whether psychosocial counselling was included in the packages; (c) whether the integration efforts extended beyond the facility level of care to the community; and (d) whether additional training elements for service providers, such as clinical communication skills or anti-stigma programmes, were included in the package. Specific details of the country integration packages are summarised in Table 1 and have been published elsewhere. $^{4,8}$

\section{Study design}

The study used a cross-sectional qualitative research design, conducting semi-structured interviews with key informants in the respective study sites to understand the obstacles, synergies and implications of efforts to scale-up a model of mental healthcare integrated into PHC. The theoretical constructs of the Consolidated Framework for Implementation Research (CFIR) framework supplemented by the WHO health systems building blocks were used to guide the analysis. ${ }^{9}$ CFIR is an overarching typology designed to promote the development and verification of implementation theory towards better understanding 'what works where and why across multiple contexts'. ${ }^{10}$ CFIR provides a group of constructs (outer setting, inner setting, individuals, characteristics of the intervention and process) that are particularly well-geared towards systematically assessing potential barriers and facilitators associated with an intervention in real-life contexts (see supplementary File 1 available at https://doi.org/10.1192/bjo.2019.7).

The six WHO health systems building blocks (service delivery; health workforce; information; medical products, vaccines and technologies; financing; and leadership and governance), ${ }^{9}$ were nested within the CFIR framework to ensure that all the essential elements of a health system were interrogated in relation to how they should be strengthened to facilitate integrated mental healthcare, particularly within the outer and inner settings of the CFIR framework.

\section{Participant selection and data-gathering}

Semi-structured qualitative interviews were conducted with provincial managers, district managers, facility managers and service providers in the six participating countries. The number and category of key stakeholders interviewed varied across countries. Face-to-face interviews with key stakeholders were conducted between May and September 2017 across the six countries following implementation of integrated packages of care in the six country district sites. A total of 121 interviews were conducted. Table 2 presents the number of interviews conducted per country.

A generic semi-structured interview guide was developed by the research team that was adapted for each country context. Guided by the WHO building blocks, it covered systems-level barriers and facilitating factors as well as implications of integrated mental healthcare as they pertained to the six building blocks (see supplementary File 2 for the interview schedule). Examples of governance and finance questions included the impact of centralised/decentralised governance structures on implementation of national policies embracing integration; intersectoral collaboration and community participation; as well as budgetary allocations to mental health and ring-fencing of the mental health budget at PHC level.

In relation to human resources, issues related to specialist staff availability and willingness to provide ongoing training and supervision within a task-sharing approach were explored; as well as the impact of integration on generalist staff; and the stability of lay counsellor cadres, where they existed. Pertaining to medicines, equipment and the infrastructure, the availability and supply of psychotropic medication was explored; as well as infrastructure for the provision of mental healthcare in PHC, especially counselling space. Concerning information systems for integrated mental health, questions related particularly to mental health indicators at PHC level, as well as data quality. Questions relating to health systems processes that enabled integration included strengthening of referral systems; processes for tracing of patients who were non-adherent; as well as shifts towards person-centred care. Trained researchers conducted audio-recorded interviews with the participants in English or the local language.

\section{Data analysis}

For data analysis, the study employed framework analysis. It is commonly used for health policy research as well as for multisite studies that have specific questions, a predetermined participant selection frame and a common set of a priori concerns being investigated. ${ }^{11}$ Analysis involves identifying commonalities and differences in the data, followed by interrogating relationships in the data, leading to descriptive or explanatory conclusions drawn around specific themes. ${ }^{11}$ Seven stages were followed: ${ }^{11}$ (a) translating data into English where necessary, and transcribing the audio-recordings into word-processing software; (b) familiarisation with the transcripts; (c) initial coding of at least three transcripts; (d) developing a working analytic framework that involved adopting the CFIR framework as a priori overarching themes in the framework matrix; (e) applying the analytic framework through coding of the transcripts; (f) charting the coded transcripts into the framework matrix through summarising the coded data; and (g) interpretation of the data. Analysis was facilitated using NVivo data management software (v. 11).

\section{Ethical considerations}

Prior to each interview, an informed consent form translated into the appropriate local language was read out to the participants. Once the participants understood the consent form and agreed to participate, they signed it and were interviewed individually.

Permission to conduct the interviews was obtained from the relevant health administrative levels for each country (Institutional Review Board of the College of Health Sciences, Addis Ababa

\begin{tabular}{|c|c|c|c|c|c|c|c|}
\hline & Ethiopia & India & Nepal & Nigeria & South Africa & Uganda & Total \\
\hline Provincial managers & 1 & 0 & 0 & 0 & 1 & 0 & 2 \\
\hline District managers & 5 & 7 & 3 & 4 & 5 & 3 & 27 \\
\hline Facility managers & 0 & 7 & 28 & 6 & 7 & 6 & 54 \\
\hline Service providers & 6 & 7 & 4 & 0 & 18 & 3 & 38 \\
\hline Total & 12 & 21 & 35 & 10 & 31 & 12 & 121 \\
\hline
\end{tabular}




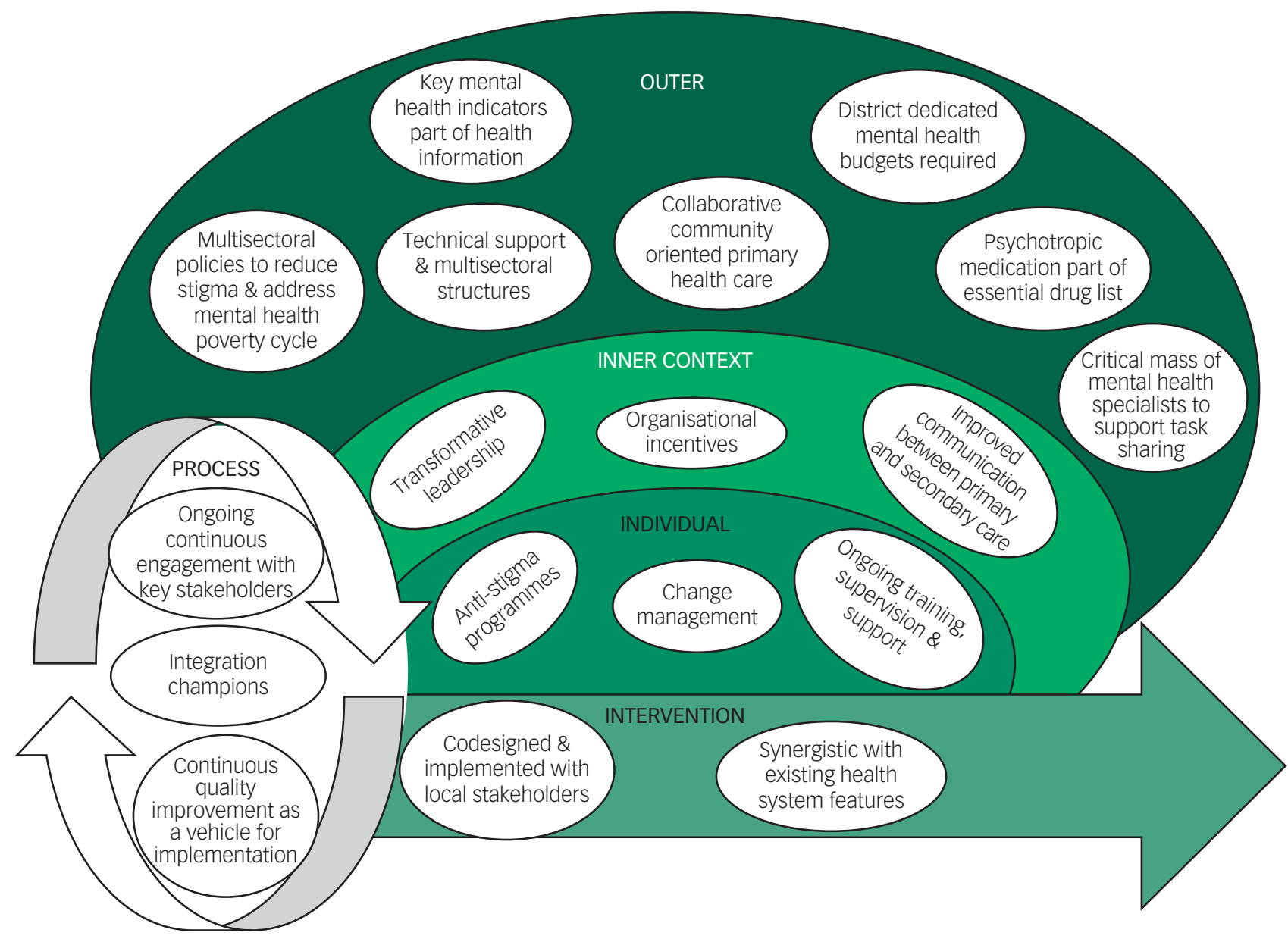

Fig. 1 Systems implications of PRogramme for Improving MEntal healthcare/Mental Health Gap Action Programme intervention efforts using the Consolidated Framework for Implementation Research framework.

University, Ref. 074/13/Psy; Public Health Foundation of India, Delhi. TRC-IEC 202.1/13; Nepal Health Research Council, Reference number 198/2015; Joint UI/UCH Ethics Committee (Nigeria); Biomedical Research Ethics Committee, University of KwaZulu-Natal, BE407/13 (South Africa), Makerere University School of Medicine Research Ethics Committee, REC REF No. 2013-143 (Uganda)).

\section{Results}

The detailed findings according to the CFIR constructs and subconstructs are presented in supplementary File 3. They are described below according to the overarching CFIR constructs and nested WHO building blocks and summarised in Fig. 1.

\section{Intervention characteristics}

Integration of mental healthcare into PHC platforms in collaboration with the research teams was perceived as beneficial for improving access to mental healthcare across the country contexts. In particular, the technical intervention guides and manuals developed were perceived to be significant primers for change:

'it [the guidelines] gives you everything, the details. You are not being afraid to diagnose and refer because it is clear.' (Facility Manager (FM) 1, South Africa).
The interventions were regarded as being contextually appropriate across the board. This was attributed to the intensive collaborative process undertaken during the formative phase, including with health ministers. This process helped ensure that the respective intervention packages were codesigned and synergistic with existing health system features and optimised the potential to strengthen existing health system structures, service delivery mechanisms and processes to absorb integrated mental healthcare. For instance, although guidelines were suggested to be beneficial, being aligned with other existing guidelines and existing training processes helped improve the goodness of fit of the innovation. Examples include strengthening of the mental health components of existing adult primary care integrated chronic care guidelines in South Africa; subsuming the mhGAP intervention guidelines in Nigeria with existing standing orders, a set of broader guidelines for use by PHC providers; ${ }^{8}$ strengthening existing referral pathways from primary to upper levels of care to include referral of mental disorders in Nigeria; mirroring a registration book used for follow-up care of patients with HIV or tuberculosis for tracking of individuals using mental health services in need of ongoing follow-up in Ethiopia; and leveraging many chronic care system processes of integrated clinical services management, an initiative to promote integrated chronic care ${ }^{12}$ in South Africa, including community health worker ward-based outreach teams for tracing patients with mental illness who were non-adherent and a chronic care dispensing medication system. 


\section{Outer setting}

Across all six countries, the findings confirmed the need for strengthening of the basic building blocks of the healthcare system at a policy level to promote integration. In relation to governance, the need for policies to strengthen multisectoral linkages in the planning, implementation and monitoring of services was emphasised. Further, the need for technical support to assist with implementation of integration policies was also emphasised. The Transcultural Psychosocial Organization in Nepal provided an example of how an externally funded organisation could assist in providing training and human resource support for integration efforts. Improved financing of integrated mental healthcare also emerged as a crucial issue, with the need for a dedicated mental health budget at PHC level identified as an unresolved policy need across all the countries. In Nepal, this was underlined as follows:

'The responsibility should be taken by the government. Only some kind of help can be taken from the donors. Government should not act like a parasite by not doing anything and simply depending on foreign donors. If we always depend on foreign donors, ... once it takes off its helping hand from us, we will be destroyed...' (Health Worker (HW) 1, Nepal)

Positive developments in relation to financing that were reported during the course of the PRIME project included the introduction of health insurance for the vulnerable in Ethiopia, which reportedly helped improve access to mental healthcare. In relation to medical products and technologies, policy changes to ensure that psychotropic medications were included on the free essential drug list were identified as essential. This materialised during the course of the PRIME project in Nepal, and was reported to improve access to treatment. With respect to the health information system, the need for policy changes to include/strengthen mental health indicators as part of the health information system was common across all the country sites. Regarding service delivery, systems re-engineering for integrated chronic care management was identified as important to enable integrated mental healthcare. In addition, there was a need for improved supply chain management for chronic medication delivery, with drug stock outs impeding continuity of medication management in Uganda, Nigeria and Nepal exemplifying this.

'The most challenge is drug stock out [sic] especially Carbamazepine. Since last year, they have not been supplying Carbamazepine.' FM2, Uganda)

In respect of 'people' who are often cited as the seventh building block of the healthcare system ${ }^{13}$ and refer to individuals, households and communities; being caught in the poverty-mental ill health cycle as well as stigma were reported to impede access to care and recovery. Policy interventions beyond the health sector are required to address this barrier, for example, population-level mental health literacy campaigns as well as social welfare and income-generation initiatives to combat poverty. The need for intersectoral governance action in the outer setting is thus highlighted. As illustrated by one participant:

'Mental health problem is economic problem [sic], it is a social problem and it is a health problem. If we try to solve the health problem who is going to solve the economic problem, who is going to solve the social problem?' (District Manager (DM) 2, Ethiopia).

\section{Inner setting}

Weaknesses in the service delivery building block played out mostly in the inner setting. In relation to infrastructure to deliver mental health services at PHC level, the lack of adequate and private spaces in health facilities for mental health consultations was highlighted across the countries, with the exception of India, where all assessments and psychosocial interventions were delivered in a separate consultation room ('Mann kaksha'). Further, although participants in most countries reported relatively acceptable internal relationships and communications at the PHC level (for instance between community health workers and nurses in South Africa; between prescribers and non-prescribers in Nepal; and between nurses and psychiatrists in India), weak feedback systems between secondary and primary levels of care were reported to impair continuity of care at PHC level, particularly in South Africa.

In terms of organisational culture, a hierarchical culture, characterised by internal maintenance with a need for stability and control, was especially dominant across most of the countries. Although it promoted control and standardisation in the provision of mental healthcare, it precluded the flexibility often required to deal with health system challenges at the coalface. 'Paper-based' leadership was identified in South Africa, Nigeria and Uganda, where upper management levels led by developing and distributing policies and plans, with little guidance given to health facilities on implementation:

'I have not been seeing the district supervising. I think the support... that we are seeing is by you people of the project and the [manager] from [referral hospital]. The district doesn't seem to be very serious on that matter.' (FM2, Uganda)

This hierarchical 'paper-based' leadership not only resulted in a paucity of technical support needed for implementation of integrated care but was also antagonistic to the promotion of the environment needed to support co-workers and managers to share workrelated emotional burdens. In South Africa, participants reported feeling powerless to solve emergent health system challenges brought about by integrated care and further reported little support for occupational emotional burdens. Greater flexibility and participation of staff in resolving challenges and obtaining support appeared to be more evident in Nepal where it was reported that frequent facility meetings were held - where anybody could participate and voice their opinions - highlighting the value of transformative leadership styles in enabling resolution of problems and coordination of services.

'We get voluntarily involved in [facility] meeting [sic]... Whenever they [managers] come to the health facility, we... ask them to let us know if there is any problem in the field... Because of all this there has been good coordination.' (HW1, Nepal)

A poor implementation climate also emerged as impeding service delivery. This was particularly the case in South Africa, where concurrent implementation of multiple interventions of different scope, size and quality led to reports of PHC staff feeling overwhelmed by so many changes in work routines, resulting in challenges in them buying-into integrated mental healthcare.

Organisational incentives and rewards that were found to promote service delivery in relation to integrated care included training and specialist support from the PRIME teams, particularly in India and Nepal. The potential of the integration packages to provide human rights-based mental healthcare was viewed as rewarding in Ethiopia, and the integrated guidelines were highlighted as simplifying comprehensive care, making patient flow more efficient and strengthening referral pathways, particularly to facility-based counsellors, in South Africa. At the time of the interviews, none of the countries were employing data-driven continuous quality improvement to incentivise implementation of integrated care, with any data collected being sent upwards within the governance structures, with very little feedback. Voluntary 
facility meetings in Nepal and compulsory meetings in Nigeria; however, created a platform for sharing information and promoting a learning climate.

\section{Characteristics of individuals}

Across all the study sites, improved self-efficacy on the part of PHC providers to deal with patients with mental disorders as a result of the interventions was reported. Notwithstanding this, there were, however, indications of stigmatising attitudes and behaviours of health workers towards people with mental disorders across all sites, exemplified by one participant referring to patients as 'lunatics and mentally derailed people' (Health Manager (HM) 1, Nigeria). Further, there were reports that integrated mental healthcare was regarded as burdensome to work routines; highlighting the need for change management to orientate healthcare staff to integrated mental health and the benefits it would bring to patients and providers. Others offered a more positive view:

'It should not be taken as a burden. ... if a farmer who is doing agriculture feels burdened to plough the field and manure the soil, then there won't be much yielding of his harvest. Likewise, health workers like us should not feel burdened even if we have to work little harder' (FM1, Nepal).

Regarding attitudes towards task-sharing - a central strategy in integrated mental healthcare - some participants highlighted that some cadres of health worker still shunned working with patients with mental illness, even after training. This was especially the case for general practitioners, particularly in Uganda, India and Nigeria. This was illustrated by a health manager based in Uganda:

'The major problem we still have is that health workers tend to think mental illness should be handled by a Psychiatric Clinical Officer or Psychiatric Nurse. So, even if they have been trained and we assume they have the knowledge, they fail to attend to the patients.' (HM1, Uganda).

Mental health specialist cadres were generally supportive of task-sharing; however, their low numbers relative to the mental health needs in communities constrained the time and effort they could spend supporting non-specialist health workers in facilities.

\section{Process}

In terms of processes of engagement, the collaborative approach with health ministry stakeholders from national to local level across all the country sites was highlighted as crucial for buy-in. In particular, facility managers were identified as important to champion integration at the coalface given their influential role over frontline workers. The importance of a change agent, in this case the technical support provided by PRIME and Nigerian mhGAP programmes, was again emphasised to drive the process of integration; as well as the need for continuous quality improvement - which engages key staff with emerging challenges and empowers them to make the necessary changes to the system to accommodate the intervention:

'...of course we have been facilitated very well by PRIME project, which has help us in many things.' (HM2, India)

\section{Discussion}

\section{Main findings}

The evidence base for the integration of mental healthcare into PHC systems of LMICs is growing rapidly, ${ }^{4}$ influenced by the Movement for Global Mental Health and a more favourable global political climate for reform. ${ }^{14}$ There is a groundswell of support emerging particularly from the increasing recognition of the role played by mental health in the achievement of Sustainable Development Goals. ${ }^{15}$ The findings from this study highlight health system synergies and obstacles that aided or obstructed ongoing mental healthcare integration efforts at district level in six LMICs as well as implications for systems strengthening required to facilitate integration.

\section{Contributors to success}

The goals of integrated mental healthcare - providing accessible, cost-effective, quality and effective mental health services to (especially) poor and disadvantaged populations - so as to break the mental ill health and poverty cycle ${ }^{16}$ received support across the board. Leveraging existing health system features and processes promoted greater synergy with the prevailing health system. A good degree of compatibility between intervention characteristics and existing policies and guidelines is a vital mechanism in health system implementation processes. ${ }^{17}$ In addition, practical implementation plans that take into account available resources is globally recognised as key to effective community mental healthcare provision. ${ }^{18}$ However, LMIC primary care systems that have historically been developed for episodic and acute care needs pose a challenge for integrated mental healthcare, which requires systems to be aligned for both acute episodes and chronic care. ${ }^{14}$ The findings of this study illustrate how existing features characteristic of chronic care systems were reportedly enabling of integration efforts. Examples include established referral pathways between PHClevel facilities and other levels of care (Nigeria), community health worker outreach teams, integrated chronic care guidelines (South Africa), a chronic medication dispensing system (South Africa), intersectoral collaborations (Nepal) and existing systems for tracking patients lost to care (Ethiopia).

\section{Obstacles}

Several health system obstacles that influenced integrated mental healthcare and strengthening of the basic building blocks of the healthcare system in these six LMICs emerged as important to create a more enabling platform for integrated care. In relation to governance, breaking the mental ill health and poverty cycle in LMICs requires a multisectoral effort; specifically, there is a need to mobilise resources outside the formal sphere of health systems to provide technical support should there be insufficient internal resources. Providing collaborative care through accessing the human capital offered by non-governmental organisations or other non-profit, community-based organisations ${ }^{19}$ should be considered and is aligned with the chronic care model. The effectiveness of integrated care packages is heavily influenced by fragmented collaboration across sectors, particularly social welfare and development (although collaboration with housing, education and justice departments all play vital roles) and applies equally to highincome settings. ${ }^{18}$

Resource constraints are a persisting feature of health systems in LMICs, and the picture in the six target countries was not different. This makes strategic resource use and multisectoral resource mobilisation all the more important. The findings of this study support the full 'horizontal' integration of mental health into existing (largely primary care) systems. Yet some injection of 'vertical' resources in the form of specialists who offer training, ongoing supervision and support, together with the requisite ring-fenced mental health budgets, emerged as essential. Further, the need for dedicated mental health champions was highlighted. The fact that this was not always evident and at times compromised in favour of other priorities speaks to the lack of dedicated, ring-fenced budgets for mental health - a crucial mechanism for stability and 
prioritisation. ${ }^{20}$ Importantly, budgets should be structured to trickle downward from national level, to local governance levels, to health facilities, following the patients who use mental health services into communities, as has been recommended globally and where investment pay-off is greatest. ${ }^{18}$

The mental health information system emerged as particularly underdeveloped across the six countries. There was an identified need to include some essential mental health indicators in the national health information system that can assist in monitoring quality of care. ${ }^{21}$ Further, the need for data to not only flow to central government databanks but to be used to provide regular feedback to local managers and health facilities for continuous quality improvement emerged as important. When routine mental healthcare indicators are not collected, there may be little incentive or motivation for busy frontline health workers to actively screen and identify common mental disorders - and offer treatment. Within quality improvement initiatives, improved health information systems have the potential to assist to promote uptake and embedding of a new intervention through assisting with change management through a process of creating 'will' to identify and find solutions to bottlenecks that emerge during service delivery - which is to be expected during the introduction of systems innovations. ${ }^{22}$ The use of continuous quality improvement to improve the coverage of prevention of mother-to-child transmission of HIV in South Africa serves as an exemplar of the change management potential of this approach. ${ }^{23}$

Persistent shortages in specialist human resources for mental health emerged as an enduring theme. Given financial constraints on the budget - against the backdrop of a substantial disease burden of mental disorders ${ }^{24}$ - scaling up of mental health through integration into PHC and task-sharing remains the only option to narrow the treatment gap. ${ }^{25,26}$ Respondents across all country sites favoured task-sharing where it was utilised as a strategy to promote integrated care. It has been shown to be cost-effective and efficient in increasing access to care for other conditions at $\mathrm{PHC}$ level, such as tuberculosis and HIV. ${ }^{25}$ However, a critical mass of specialist human resources is required to provide supervision and support. Task-sharing included sharing of specialist tasks with community health workers across all the country sites. There is international evidence that with adequate training and support this level of worker can successfully be used to help screen and identify people with mental disorders, as well as deliver certain evidencebased psychosocial counselling interventions. ${ }^{20,26}$ Nonetheless, we should proceed with caution, as community health workers can very easily become overburdened in similar ways to PHC-level health workers, especially in settings with substantial burdens of disease. Governing and supporting the tasks of community health workers strategically is key.

\section{Limitations}

Some limitations should be considered. The study does not include the voices of patients with mental illnesses, their families and caretakers nor service providers operating outside the ministries of health - such as community-based organisations. To create a degree of standardisation for the analysis of the interviews, they had to be translated into English where necessary. It might be possible that some of the context was lost during this process, although the close involvement of respective country partners during transcription and translation processes somewhat reduced this risk.

\section{Implications}

To conclude, the findings of this study indicate that the scale-up of integrated mental healthcare into PHC in LMICs is complex, requiring more than just training of existing PHC providers, historically the approach taken to integrate mental healthcare. The need to strengthen the basic building blocks of the healthcare system to create a more enabling platform for integrated mental healthcare emerged as paramount for successful integration. In light of the changing disease burden from predominantly acute conditions towards greater numbers of chronic conditions, healthcare systems in LMICs are increasingly engaging in healthcare system redesign from vertical programming for acute conditions, towards the provision of integrated care for chronic ones. These health system reforms should provide a more enabling platform that can be leveraged for the integration of mental healthcare. Advocates of global mental health should take heed of the need for these broader health system reforms to create an enabling context for integrated mental healthcare. There needs to be a balance between the focus on mental health only, with the need for integrated care as a whole, so as not to have the unintended consequence of reinforcing vertical programming for mental health.

Inge Petersen, Research Professor and Director, Centre for Rural Health, School of Nursing and Public Health, University of KwaZulu-Natal, South Africa; André van Rensburg, Senior Researcher, Centre for Rural Health, School of Nursing and Public Health, University of KwaZulu-Natal, South Africa; Fred Kigozi, Senior Consultant Psychiatrist/ Researcher, Butabika National Referral and Teaching Mental Hospital, Uganda; Maya Semrau, Research Fellow, Centre for Global Mental Health, Department of Health Service and Population Research, Institute of Psychiatry, Psychology and Neuroscience, King's College London; and Global Health and Infection Department, Brighton and Sussex Medical School, UK; Charlotte Hanlon, Adjunct Associate Professor, Department of Psychiatry, Addis Ababa University, Ethiopia; and Reader in Global Mental Health, Centre for Global Mental Health, Health Service and Population Research Department, Institute of Psychiatry, Psychology and Neuroscience, King's College London, UK; Jibril Abdulmalik, Senior Lecturer, Department of Psychiatry, University of Ibadan, Nigeria; Lola Kola, Researcher, Department of Psychiatry, University of Ibadan, Nigeria; Abebaw Fekadu, Professor in Global Mental Health, Centre for Innovative Drug Development and Therapeutic Trials for Africa (CDT-Africa), College of Health Sciences, Addis Ababa University, Ethiopia; and Global Health \& Infection Department, Brighton and Sussex Medical School, UK; Oye Gureje, Professor of Psychiatry and Director, WHO Collaborating Centre for Research and Training in Mental Health, Neurosciences and Substance Abuse, Department of Psychiatry, University of Ibadan, Nigeria; and Professor Extraordinary, Department of Psychiatry, Stellenbosch University, South Africa; Dristy Gurung, Research Coordinator, Transcultural Psychosocial Organisation, Nepal; Mark Jordans (ID) Reader, Center for Global Mental Health, King's College London, UK; Ntokozo Mntambo, Researcher, Centre for Rural Health, School of Nursing and Public Health, University of KwaZulu-Natal, South Africa; James Mugisha, Senior Lecturer, Department of Sociology, Kyambogo University and Butabika National Referral and Teaching Mental Hospital, Uganda; Shital Muke, Researcher, Sangath, India; Ruwayda Petrus, Senior Lecturer, Department of Psychology, School of Applied Human Sciences, University of KwaZulu-Natal, South Africa; Rahul Shidhaye, Clinical Psychiatrist, Public Health Foundation of India and CAPHRI School for Public Health and Primary Care, Maastricht University, the Netherlands; Joshua Ssebunnya, Researcher, Butabika National Referral and Teaching Mental Hospital, Uganda; Bethlehem Tekola, Research Associate, Health Service \& Population Research, Institute of Psychiatry, Psychology and Neuroscience, King's College London, UK; Nawaraj Upadhaya, Researcher, Transcultural Psychosocial Organisation, Nepal; Vikram Patel, Professor, Department of Global Health and Social Medicine, Harvard Medical School and Harvard TH Chan School of Public Health, Harvard University, USA; and Sangath and the Public Health Foundation of India, India; Crick Lund (D), Professor of Public Mental Health, Alan J Flisher Centre for Public Mental Health, Department of Psychiatry and Mental Health, University of Cape Town, South Africa; and Professor of Global Mental Health and Development, Centre for Global Mental Health, Department of Health Service and Population Research, Institute of Psychiatry, Psychology and Neuroscience, King's College London, UK: Graham Thornicroft, Professor of Community Psychiatry, Centre for Global Mental Health and Centre for Implementation Science, Institute of Psychiatry, Psychology and Neuroscience, King's College London, UK

Correspondence: Inge Petersen, Centre for Rural Health, School of Nursing and Public Health, University of KwaZulu-Natal, South Africa. E-mail: peterseni@ukzn.ac.za

First received 28 Aug 2018, final revision 21 Jan 2019, accepted 23 Jan 2019

\section{Funding}

The research leading to these results is funded by the European Union's Seventh Framework Programme (FP7/2007-2013) under grant agreement number 305968. The funder had no role in study design, data collection and analysis, decision to publish or preparation of the manuscript. It is also an output of the PRogramme for Improving Mental health carE (PRIME) supported by the UK Department for International Development (201446). The views expressed do not necessarily reflect the UK Government's official policies. The funder did not have any involvement in the study design, collection, analysis or interpretation of data or writing of the manuscript. G.T. is supported by the National Institute for Health Research (NIHR) Collaboration for Leadership in Applied Health Research and Care (CLAHRC) South London and by the NIHR Applied Research Centre (ARC) at King's College London NHS Foundation Trust, and the NIHR Applied Research and the NIHR Asset Global Health Unit award. The 
views expressed are those of the author(s) and not necessarily those of the NHS, the NIHR or the Department of Health and Social Care. G.T. receives support from the National Institute of Mental Health of the National Institutes of Health under award number R01MH100470 (Cobalt study). G.T. is supported by the UK Medical Research Council in relation the Emilia (MR/ S001255/1) and Indigo Partnership (MR/R023697/1) awards. I.P. and G.T. also receive support from the National Institute of Mental Health of the National Institutes of Health under award number R01MH100470. I.P., A.v.R., C.H. and C.L. are funded by the NIHR Global Health Research Unit on Health System Strengthening in Sub-Saharan Africa, King's College London (GHRU 16/136/54) using UK aid from the UK Government The views expressed in this publication are those of the authors and not necessarily those of the NIHR or the Department publicaand Social Care. $\mathrm{C}$. H. additionally receives support from AMARI as part of the DELTAS Africa Initiative (DEL-15-01)

\section{Acknowledgements}

The partner organisations involved in Emerald are Addis Ababa University (AAU), Ethiopia; Butabika National Mental Hospital (BNH), Uganda; Gesellschaft für Ablauforganisation :milliarium GmBH \& Co. KG (GABO:mi), Germany; HealthNet TPO, Netherlands; King's College London (KCL), UK: Public Health Foundation of India (PHFI), India; Transcultural Psychosocial Organization Nepal (TPO Nepal), Nepal; Universidad Autonoma de Madrid (UAM), Spain: University of Cape Town (UCT), South Africa; University of Ibadan (UI), Nigeria; University of KwaZulu-Natal (UKZN), South Africa; and the World Health Organization (WHO), Switzerland.

The Emerald programme is led by Professor Graham Thornicroft at KCL. The project coord ination group consists of Professor Atalay Alem (AAU), Professor José Luis Ayuso-Mateos (UAM), Dr Dan Chisholm (WHO), Dr Stefanie Fülöp (GABO:mi), Professor Oye Gureje (UI), Dr Charlotte Hanlon (AAU; KCL), Dr Mark Jordans (HealthNet TPO; TPO Nepal; KCL), Dr Fred Kigozi (BNH), Professor Crick Lund (UCT), Professor Inge Petersen (UKZN), Dr Rahul Shidhaye (PHFI), Professor Graham Thornicroft (KCL) and Ms Nicole Votruba (GABO:mi).

Parts of the programme are also coordinated by Ms Shalini Ahuja (PHFI), Dr Jibril Omuya Abdulmalik (UI), Ms Kelly Davies (KCL), Ms Sumaiyah Docrat (UCT), Dr Catherine Egbe (UKZN), Dr Sara Evans-Lacko $(\mathrm{KCL})$, Dr Margaret Heslin $(\mathrm{KCL})$, Dr Dorothy Kizza $(\mathrm{BNH})$, Dr Lola Kola (UI), Dr Heidi Lempp (KCL), Dr Pilar López (UAM), Ms Debra Marais (UKZN), Ms Blanca Mellor (UAM), Mr Durgadas Menon (PHFI), Dr James Mugisha (BNH), Ms Sharmishtha Nanda (PHFI), Dr Anita Patel $(K C L)$, MS Shoba Raja (BasicNeeds, India; $K C L$ ), Dr Maya Semrau (KCL), Mr Joshua Ssebunya (BNH), Mr Yomi Taiwo (UI) and Mr Nawaraj Upadhaya (TPO Nepal).

The Emerald programme's scientific advisory board includes A/Professor Susan Cleary (UCT), Dr Derege Kebede (WHO, Regional Office for Africa), Professor Harry Minas (University of Melbourne, Australia), Mr Patrick Onyango (TPO Uganda), Professor Jose Luis Salvador Carulla (University of Sydney, Australia) and Dr R Thara (Schizophrenia Research Foundation (SCARF), India).

The following individuals are members of the Emerald consortium: Dr Kazeem Adebayo (UI), Ms Jennifer Agha (KCL), MS Ainali Aikaterini (WHO), Dr Gunilla Backman (London School of Hygiene \& Tropical Medicine; KCL), Mr Piet Barnard (UCT), Dr Harriet Birabwa (BNH), MS Erica Breuer (UCT), Mr Shveta Budhraja (PHFI), Amit Chaturvedi (PHFI), Mr Daniel Chekol (AAU), Mr Naadir Daniels (UCT), Mr Bishwa Dunghana (TPO Nepal), Ms Gillian Hanslo (UCT), Ms Edith Kasinga (UCT), Ms Tasneem Kathree (UKZN), Mr Suraj Koirala (TPO Nepal), Professor Ivan Komproe (HealthNet TPO), Dr Mirja Koschorke (KCL), Ann Uustalu (European Commission) Mr Nagendra Luitel (TPO Nepal), Dr David MCDaid (KCL) Ms Immaculate Nantongo (BNH), Dr Sheila Ndyanabangi (BNH), Dr Bibilola Oladeji (UI), Professor Vikram Patel Nantongo (BNH), Dr Sheila Ndyanabangi (BNH), Dr Bibilola Oladeji (Ul), Professor Vikram Patel
$(\mathrm{KCL})$, Ms Louise Pratt $(\mathrm{KCL})$, Professor Martin Prince (KCL), Ms M Miret (UAM), Ms Warda Sablay (UCT), Mr Bunmi Salako (UI), Dr Tatiana Taylor Salisbury (KCL), Dr Shekhar Saxena (WHO), Ms One Selohilwe (UKZN), Dr Ursula Stangel (GABO:mi), Professor Mark Tomlinson (UCT), Dr Abebaw Fekadu (AAU) and Ms Elaine Webb (KCL).

\section{Supplementary material}

Supplementary material is available online at https://doi.org/10.1192/bjo.2019.7

\section{References}

1 Keynejad RC, Dua T, Barbui C, Thornicroft G. WHO Mental Health Gap Action Programme (mhGAP) Intervention Guide: a systematic review of evidence from low and middle-income countries. Evid Based Ment Health 2018; 21: 30-4.

2 Patel V, Chisholm D, Parikh R, Charlson FJ, Degenhardt L, Dua T, et al. Addressing the burden of mental, neurological, and substance use disorders: key messages from Disease Control Priorities, 3rd edition. Lancet 2016; 387 1672-85.

3 Singla DR, Kohrt BA, Murray LK, Anand A, Chorpita BF, Patel V. Psychological treatments for the world: lessons from low- and middle-income countries. Annu Rev Clin Psychol 2017; 13: 149-81.

4 Lund C, Tomlinson M, Patel V. Integration of mental health into primary care in low- and middle-income countries: the PRIME mental healthcare plans. $\mathrm{Br} J$ Psychiatry 2016; 208 (suppl 56): s1-3.
5 Thornicroft G, Semrau M. Mental health capacity building in low and middle income countries: the Emerald Programme. Epidemiol Psychiatr Sci 2018; 27: $1-2$.

6 Mugisha J, Abdulmalik J, Hanlon C, Petersen I, Lund C, Upadhaya N, et al. Health systems context(s) for integrating mental health into primary health care in six Emerald countries: a situation analysis. Int J Ment Health Syst 2017; 11: 7.

7 World Health Organization. mhGAP Intervention Guide for Mental, Neurological and Substance Use Disorders in Non-Specialized Health Settings. World Health Organization, 2010

8 Abdulmalik J, Kola L, Fadahunsi W, Adebayo K, Yasamy MT, Musa E, et al. Country contextualization of the mental health gap action programme intervention guide: a case study from Nigeria. PLOS Med 2013; 10: e1001501.

9 World Health Organization. Everybody's Business - Strengthening Health Systems to Improve Health Outcomes: WHO's Framework for Action. World Health Organization, 2007.

10 Damschroder $\mathrm{J}$, Aron DC, Keith RE, Kirsh SR, Alexander JA, Lowery JC. Fostering implementation of health services research findings into practice: a consolidated framework for advancing implementation science. Implement Sci 2009; 4: 50 .

11 Gale NK, Heath G, Cameron E, Rashid S, Redwood S. Using the framework method for the analysis of qualitative data in multi-disciplinary health research. BMC Med Res Methodol 2013; 13: 117.

12 Department of Health, Republic of South Africa. Ideal Clinic Manual version 18. Department of Health, 2018.

13 GAVI. What are the Health System Building Blocks? GAVI, 2013

14 Patel V, Belkin GS, Chockalingam A, Cooper J, Saxena S, Unützer J. Grand challenges: integrating mental health services into priority health care platforms. PLOS Med 2013; 10: e1001448.

15 Patel V, Saxena S, Lund C, Thornicroft G, Baingana F, Bolton P, et al. The Lancet Commission on global mental health and sustainable development. Lancet 2018; 392: 1553-98.

16 Lund C, De Silva M, Plagerson S, Cooper S, Chisholm D, Das J, et al. Poverty and mental disorders: breaking the cycle in low-income and middle-income countries. Lancet 2011; 378: 1502-14

17 May CR, Mair F, Finch T, MacFarlane A, Dowrick C, Treweek S, et al. Development of a theory of implementation and integration: normalization process theory. Implement Sci 2009; 4: 29.

18 Thornicroft G, Alem A, Antunes Dos Santos R, Barley E, Drake RE, Gregorio G, et al. WPA guidance on steps, obstacles and mistakes to avoid in the implementation of community mental health care. World Psychiatry 2010; 9: 67-77.

19 Jordans MJD, Luitel NP, Pokhrel P, Patel V. Development and pilot testing of a mental healthcare plan in Nepal. Br J Psychiatry 2016; 208 (suppl 56): s21-8.

20 Petersen I, Marais D, Abdulmalik J, Ahuja S, Alem A, Chisholm D, et al. Strengthening mental health system governance in six low- and middle-income countries in Africa and South Asia: challenges, needs and potential strategies. Health Policy Plann 2017; 32: 699-709.

21 Crick L. Improving quality of mental health care in low-resource settings: lessons from PRIME. World Psychiatry 2018; 17: 47-8.

22 Horwood C, Butler L, Barker P, Phakathi S, Haskins L, Grant M, et al. A continuous quality improvement intervention to improve the effectiveness of community health workers providing care to mothers and children: a cluster randomised controlled trial in South Africa. Hum Resour Health 2017; 15: 39.

23 Doherty T, Chopra M, Nsibande D, Mngoma D. Improving the coverage of the PMTCT programme through a participatory quality improvement intervention in South Africa. BMC Public Health 2009; 9: 406.

24 Whiteford HA, Ferrari AJ, Degenhardt L, Feigin V, Vos T. The global burden of mental, neurological and substance use disorders: an analysis from the Global Burden of Disease Study 2010. PloS One 2015; 10: e0116820.

25 Seidman G, Atun R. Does task shifting yield cost savings and improve efficiency for health systems? A systematic review of evidence from low-income and middle-income countries. Hum Resour Health 2017; 15: 29.

26 Eaton J, McCay L, Semrau M, Chatterjee S, Baingana F, Araya R, et al. Scale up of services for mental health in low-income and middle-income countries. Lancet 2011; 378: 1592-603. 